\title{
Alterações dermatológicas em pacientes oncológicos - adultos e crianças
}

Dermatological alterations in oncological patients - adults and children

Dolores Gonzalez Fabra', Ana Paula Kayo², Andrea Regina de Oliveira Leal', Vanessa Salzano³, Flávia Guglielmino²

\begin{abstract}
Resumo
0 tratamento oncológico, seja cirúrgico e/ou quimioterápico e/ou radioterápico, acarreta inúmeros efeitos adversos cutâneos - muitos deles já relatados na literatura. Por meio do Ambulatório de Reabilitação Dermato-Cosmiátrica da Faculdade de Medicina do ABC, pudemos catalogar as alterações dermatológicas e cosmiátricas decorrentes do tratamento oncológico, além de tratá-las durante a vigência do tratamento, sem interferir em seus resultados. 0 objetivo deste trabalho foi dividir as alterações dermatológicas e cosmiátricas decorrentes do tratamento oncológico em três grandes grupos: alterações desencadeadas, agravadas e não relacionadas ao tratamento oncológico - classificação esta ainda inédita na literatura médica. Além disso, o trabalho classificou as alterações de acordo com o tratamento oncológico realizado.
\end{abstract}

Palavras-chave: Efeitos adversos; neoplasia; radioterapia; quimioterapia.

\section{Abstract}

Cancer treatment, surgery and/or chemotherapy and/or radiotherapy, involves numerous adverse effects on the skin. Most of these effects have already been reported in the literature. The Ambulatory of Dermatocosmiatry Rehabilitation from Faculdade de Medicina do ABC catalogued cosmetics alterations and injuries on the skin due to cancer treatment, besides treating them during therapy without interfering in the clinical results. The objective of this work was to divide the dermatologic injuries and cosmetic skin changes resulting from cancer treatment in three broad groups: changes induced by treatment, enhanced by treatment and non-related to it. This is the first report in medical literature. Besides, the study characterized the changes in accordance to the oncological treatment made.

Keywords: Adverse effects; neoplasm; radiotherapy; drug therapy.

Recebido: 03/03/2008

Revisado: 08/05/2009

Aprovado: 08/05/2009

\footnotetext{
Disciplina de Dermatologia da Faculdade de Medicina do ABC (FMABC), Santo André (SP), Brasil

2 Departamento de Clínica Médica da FMABC, Santo André (SP), Brasil

${ }^{3}$ Departamento de Dermatologia do Hospital Infantil Darcy Vargas, São Paulo (SP), Brasil

Endereço para correspondência: Dolores Gonzalez Fabra - Faculdade de Medicina do ABC - Avenida Príncipe de Gales, 821 - Príncipe de Gales -

CEP 09060-650 - Santo André (SP), Brasil - Tel: 3885-9020 - Fax: 3052-0610 - E-mail: doloresgonzalezfabra@uol.com.br
} 


\section{Introdução}

A grande diversidade de tratamentos oncológicos propostos aos pacientes com câncer eleva em muito sua sobrevida. Desta forma, é cada vez maior o número de pacientes que apresentam complicações dermatológicas relacionadas à própria patologia ou ao tratamento a ela proposto.

Tanto o tratamento cirúrgico, como o tratamento quimioterápico e radioterápico estão implicados no aparecimento de inúmeros efeitos colaterais ${ }^{1}$.

A pele, os cabelos, as unhas e as mucosas são estruturas que podem apresentar alterações decorrentes do estado fisiológico do paciente. Podem ainda sofrer influências de doenças sistêmicas, tumores internos e dos tratamentos antineoplásicos ${ }^{2}$.

A exérese cirúrgica do tumor pode acarretar sequelas locais, tanto cosméticas como funcionais, tais como: cicatrizes atróficas, hipertróficas e queloidianas, hiperpigmentação, fibrose, etc.

As reações cutâneas desencadeadas por quimioterápicos não são raras, mas apresentam difícil manejo ${ }^{3}$. As drogas quimioterápicas atuam sobre as células que apresentam alta taxa de multiplicação ${ }^{4}$, agindo não somente sobre as células neoplásicas, mas também sobre qualquer célula do organismo com alto metabolismo. Desta maneira, a pele e seus anexos representam um dos maiores alvos do tratamento quimioterápico ${ }^{5}$.

O principal mecanismo de ação dos quimioterápicos é induzir a morte celular através da apoptose ${ }^{6}$, sendo que os efeitos sobre o tecido cutâneo são, na maior parte, decorrentes de reações tóxicas e não alérgicas ${ }^{7}$.

$\mathrm{Na}$ maioria dos casos, os pacientes recebem doses elevadas de múltiplas drogas quimioterápicas ${ }^{8}$, e o espectro de doenças cutâneas decorrentes deste tratamento nos pacientes oncológicos difere em muito das dermatopatias desenvolvidas pelos pacientes imunossuprimidos por outras etiologias?.

A radioterapia ocasiona inúmeros efeitos sobre o tecido cutâneo, variando entre agudos (até seis meses após o início da terapia) ou crônicos (após seis meses do início da terapia) e leve, moderado ou grave, dependendo da localização, tamanho e profundidade do tumor irradiado $^{10}$. Nos casos leves, pode ocorrer um discreto eritema e nos casos graves, pode haver necrose cutânea com risco aumentado de úlceras crônicas e degeneração maligna ${ }^{11}$.

A aparição das complicações a curto, médio ou longo prazo é, sem dúvida, um dos principais fatores limitantes do tratamento antineoplásico e, por meio da sua descrição, é possível formular estratégias apropriadas para melhor conduzi-las.

Os efeitos colaterais decorrentes do tratamento quimio/radioterápico e/ou cirúrgico acarretam importantes consequências na qualidade de vida desses pacientes sendo, portanto, necessário um acompanhamento multidisciplinar. Tal acompanhamento é necessário, principalmente entre os oncologistas e os dermatologistas, para prevenir, minimizar e melhor entender esses efeitos adversos dos tratamentos oncológicos ${ }^{12}$.
Foi com este objetivo que instituímos o Ambulatório de Reabilitação Dermato-cosmiátrica da Faculdade de Medicina do ABC (FMABC). Trata-se de um projeto pioneiro de assistência integral aos pacientes oncológicos, realizado em conjunto entre as Disciplinas de Dermatologia e Oncologia da FMABC, além da colaboração de outras especialidades, tais como a Cirurgia Plástica, Psiquiatria, Fisioterapia, entre outras.

Os pacientes oncológicos que serão submetidos a tratamento quimio/radioterápico e/ou cirúrgico na FMABC passam previamente pelo Ambulatório de Reabilitação, onde recebem orientações preventivas dermato-cosmiátricas a fim de minimizar os efeitos colaterais e sequelas do tratamento oncológico. Quando iniciado o tratamento proposto para cada paciente, este é reavaliado quinzenalmente para acompanhamento e tratamento dos possíveis efeitos colaterais dermato-cosmiátricos decorrentes da quimio e/ou radioterapia e/ou cirurgia.

O objetivo deste trabalho é catalogar as alterações dermatológicas e cosmiátricas desenvolvidas pelos pacientes oncológicos (adultos e crianças) atendidos no Ambulatório de Reabilitação Dermato-Cosmiátrica da FMABC, independentemente do tratamento oncológico proposto (quimio e/ou radioterápico e/ou cirúrgico), subdividindo-as em três grupos: alterações dermatológicas e cosmiátricas desencadeadas, agravadas e não relacionadas ao tratamento oncológico.

\section{Casuística e métodos}

Instituímos o Ambulatório a partir de novembro de 2004 para agendamento no ano corrente de 2005 e iniciamos o atendimento à população geral apenas a partir de janeiro de 2005. Foram atendidos 229 pacientes no período de janeiro de 2005 a junho de 2006, sendo realizados 765 atendimentos entre consultas e retornos desses pacientes.

Os pacientes atendidos eram provenientes do Ambulatório de Oncologia da FMABC e, aberto para pacientes de qualquer sexo, idade, tipo de tumor diagnosticado e tratamento oncológico proposto.

Os 240 pacientes atendidos nesse período foram avaliados quanto ao aparecimento de dermatopatias durante o tratamento oncológico, todas elas catalogadas e tratadas, além de separadas em dois grandes grupos, levando-se em conta a idade do paciente. As dermatopatias foram, portanto, divididas entre alterações dermatológicas desenvolvidas pelos pacientes maiores de 16 anos e os menores de 16. Estas, por sua vez, foram subdivididas em três subgrupos: alterações desencadeadas, agravadas ou não relacionadas ao tratamento quimio e/ou radioterápico e/ou cirúrgico.

Este estudo foi aprovado pelo Comitê de Ética em Pesquisa da FMABC (registro 54/2008).

\section{Resultados}

Dos 240 pacientes atendidos no Ambulatório de Reabilitação Dermato-Cosmiátrica da FMABC, 229 eram maiores de 16 anos, sendo 
os 11 pacientes restantes menores de 16 anos. As alterações dermatológicas desenvolvidas pelos pacientes maiores de 16 anos atendidos neste ambulatório foram catalogadas e somaram um total de 59 dermatopatias decorrentes do tratamento oncológico - quimio e/ou radioterápico e/ou cirúrgico. As alterações dermatológicas e cosmiátricas desenvolvidas pelos pacientes maiores de 16 anos foram subdivididas em três subgrupos: alterações dermatológicas desencadeadas, agravadas e não relacionadas ao tratamento quimio e/ou radioterápico e/ou cirúrgico (Tabela 1).

Dentre as alterações dermatológicas e cosmiátricas desencadeadas pelo tratamento e presentes nos pacientes maiores de 16 anos, a mais prevalente foi a alteração pilosa incluindo eflúvio telógeno/anágeno e alopecia, seguido por xerose cutânea, correspondendo a 60 (22,7\%) e 56 (21,2\%) diagnósticos. A radiodermite se desenvolveu em 19 pacientes $(7,1 \%)$, a alteração da cor das unhas foi apresentada em 16 pacientes (6,0\%), o mesmo número correspondendo ao diagnóstico de queilite; segue-se a rarefação da pilificação do corpo e candidíase, ambos com $11(4,1 \%)$ casos. Apresentaram linfedema dez pacientes (3,7\%) e nove (3,4\%) onicólise. Rarefação de sobrancelhas, erisipela e hipercromia pós-quimioterapia corresponderam a oito $(3,0 \%)$ casos cada. Houve seis $(2,2 \%)$ pacientes que apresentaram prurigo e seis $(2,2 \%)$ com alteração das mucosas; quatro $(1,5 \%)$ pacientes desenvolveram pilificação na área da reconstrução cirúrgica. Dermatite pigmentar linear, xerostomia e limitação do movimento dos membros superiores corresponderam a três casos cada $(1,1 \%)$. Escabiose, queimadura pós-radioterapia e granuloma de corpo estranho na cicatriz cirúrgica foram verificados em dois pacientes cada $(0,7 \%)$. Apenas uma paciente $(0,3 \%)$ apresentou ausência de mamilo.

A principal alteração dermatológica e cosmiátrica agravada durante o tratamento oncológico e presente nos pacientes maiores de 16 anos foi melasma com 40 (25,3\%) diagnósticos, seguida por $36(22,7 \%)$ casos de onicomicose, $22(13,9 \%)$ de tinha dos pés/mãos/corpo, $18(11,3 \%)$ pacientes apresentaram dermatite seborreica, $15(9,4 \%)$ cicatriz hipertrófica/queloide, 7 (4,4\%) foliculite. Verruga vulgar e paroníquia foram diagnosticados em $6(3,7 \%)$ pacientes cada, $5(3,1 \%)$ casos de hiperqueratose plantar foram diagnosticados. Apenas um (0,6\%) diagnóstico de pitiríase versicolor, olheiras e psoríase foi realizado.

Dentre as alterações dermatológicas e cosmíatricas não relacionadas ao tratamento oncológico e presentes nos pacientes maiores de 16 anos, a mais frequente foi queratose actínica com 13 (18,5\%) casos, seguida de $12(17,1 \%)$ casos de melanose solar, 9 (12,8\%) dermatite de contato, 7 (10,0\%) fotoenvelhecimento, 3 (4,2\%) casos de acrocórdons e dermatite ocre, 2 (2,8\%) de lipodistrofia, rosácea, telangiectasias e carcinoma basocelular e 1 (1,4\%) diagnóstico de pitiríase alba, úlce-

Tabela 1 - Alterações dermatológicas e cosmiátricas e o tratamento oncológico dos pacientes maiores de 16 anos atendidos no Ambulatório

\begin{tabular}{|c|c|c|c|c|c|}
\hline $\begin{array}{l}\text { Alterações desencadeadas pelo } \\
\text { tratamento }\end{array}$ & $\begin{array}{c}\text { Número de } \\
\text { pacientes (\%) }\end{array}$ & $\begin{array}{l}\text { Alterações agravadas pelo } \\
\text { tratamento }\end{array}$ & $\begin{array}{c}\text { Número de } \\
\text { pacientes (\%) }\end{array}$ & $\begin{array}{l}\text { Alterações não relacionadas } \\
\text { ao tratamento }\end{array}$ & $\begin{array}{c}\text { Número de } \\
\text { pacientes (\%) }\end{array}$ \\
\hline Eflúvio telógeno/anágeno/alopecia & $60(22,7)$ & Melasma & $40(25,3)$ & Queratose actínica & $13(18,5)$ \\
\hline Xerose cutânea & $56(21,2)$ & Onicomicose & $36(22,7)$ & Melanose solar & $12(17,1)$ \\
\hline Radiodermite & $19(7,1)$ & Tinha pés/mãos/corpo & $22(13,9)$ & Dermatite de contato & $9(12,8)$ \\
\hline Alteração da cor das unhas & $16(6,0)$ & Dermatite seborreica & $18(11,3)$ & Efélides & $7(10,0)$ \\
\hline Queilite & $16(6,0)$ & Cicatriz hipertrófica/queloide & $15(9,4)$ & Fotoenvelhecimento & $5(7,1)$ \\
\hline Rarefação da pilificação do corpo & $11(4,1)$ & Foliculite & $7(4,4)$ & Acrocórdons & $3(4,2)$ \\
\hline Candidíase & $11(4,1)$ & Verruga vulgar & $6(3,7)$ & Dermatite ocre & $3(4,2)$ \\
\hline Linfedema & $10(3,7)$ & Paroníquia & $6(3,7)$ & Lipodistrofia & $2(2,8)$ \\
\hline Onicólise & $9(3,4)$ & Hiperqueratose plantar & $5(3,1)$ & Rosácea & $2(2,8)$ \\
\hline Rarefação das sobrancelhas & $8(3,0)$ & Pitiríase versicolor & $1(0,6)$ & Telangiectasias & $2(2,8)$ \\
\hline Erisipela & $8(3,0)$ & Olheiras & $1(0,6)$ & Carcinoma basocelular & $2(2,8)$ \\
\hline Hipercromia pós-quimioterapia & $8(3,0)$ & Psoríase & $1(0,6)$ & Pitiríase alba & $1(1,4)$ \\
\hline Alteração das mucosas & $6(2,2)$ & & & Ùlceras & $1(1,4)$ \\
\hline Prurigo & $6(2,2)$ & & & Pediculose & $1(1,4)$ \\
\hline Pifilicação na área da reconstrução & $4(1,5)$ & & & Vasos varicosos & $1(1,4)$ \\
\hline Dermatite pigmentar linear & $3(1,1)$ & & & Nevos melanocíticos & $1(1,4)$ \\
\hline Xerostomia & $3(1,1)$ & & & Queratodermia dos glúteos & $1(1,4)$ \\
\hline Limitação do movimento dos mmss & $3(1,1)$ & & & Queratose seborreica & $1(1,4)$ \\
\hline Escabiose & $2(0,7)$ & & & Rítides & $1(1,4)$ \\
\hline Queimadura pós- radioterapia & $2(0,7)$ & & & Neurilemoma & $1(1,4)$ \\
\hline Granuloma de CE na cicatriz cirúrgica & $2(0,7)$ & & & Leucodermia puntacta & $1(1,4)$ \\
\hline Ausência de mamilo & $1(0,3)$ & & & & \\
\hline
\end{tabular}


ras, pediculose, vasos varicosos, nevos melanocíticos, queratodermia dos glúteos, queratose seborreica, rítides, neurilemoma e leucodermia puntacta.

O segundo grande grupo, composto pelos pacientes menores de 16 anos, também tiveram suas alterações dermatológicas e cosmiátricas desenvolvidas durante o tratamento quimio e/ou radioterápico e/ou cirúrgico subdivididas em dois subgrupos: alterações dermatológicas desencadeadas e agravadas (Tabela 2).

Dentre as alterações dermatológicas e cosmiátricas desencadeadas pelo tratamento oncológico e presentes nos pacientes menores de 16 anos, a mais prevalente foi a xerose cutânea (5 pacientes - 41,6\%), seguida pela alteração pilosa (eflúvio telógeno/anágeno e alopecia) com 3 (25,0\%) diagnósticos, além de prurido, hipercromia no local da cicatriz cirúrgica, dermatite seborreica e estrias com 1 (8,3\%) diagnóstico cada.

Dentre as alterações dermatológicas e cosmiátricas agravadas pelo tratamento oncológico e presentes nos pacientes menores de 16 anos, a mais frequente foi a pitiríase alba, com $4(57,1 \%)$ casos seguida por uma $(14,2 \%)$ queixa de olheira, acne e dermatite atópica.

Correlacionando as alterações dermato-cosmiátricas com o tipo de tratamento oncológico proposto (Tabela 3), pudemos perceber que a principal alteração desencadeada pela exérese cirúrgica do tumor foi o linfedema (32\%), seguida por alteração capilar (eflúvio telógeno/anágeno/alopecia) e pilificação na área de reconstrução (14\%), candidíase e limitação do movimento dos membros superior (11\%), granuloma de corpo estranho no local da ferida operatória (7\%) e escabiose, ausência de mamilo e hipercromia no local da cicatriz cirúrgica (4\%).

Dentre as lesões decorrentes da quimioterapia, a mais prevalente foi alteração capilar (eflúvio telógeno/anágeno/alopecia) (28\%), xerose (25\%), alteração da cor das unhas (8\%), rarefação da pilificação do corpo $(5 \%)$, queilite $(4,8 \%)$, onicólise $(4,3 \%)$, candidíase, rarefação de sobrancelhas e hipercromia pós-quimioterapia $(3,8 \%)$, erisipela $(3,3 \%)$, prurigo $(2,8 \%)$, alteração das mucosas $(2,4 \%)$, dermatite pigmentar linear e xerostomia $(1,4 \%)$, escabiose, prurido, dermatite seborreica e estrias $(0,4 \%)$.

Tabela 2 - Alterações dermatológicas e cosmiátricas e o tratamento oncológico dos pacientes menores de 16 anos atendidos no Ambulatório

\begin{tabular}{lclc}
\hline Alterações desencadeadas pelo tratamento & Número de pacientes (\%) & Alterações agravadas pelo tratamento & Número de pacientes $(\%)$ \\
\hline Xerose cutânea & $5(41,6)$ & Pitiríase Alba & $4(57,1)$ \\
Eflúvio telógeno/anágeno/alopecia & $3(25,0)$ & Olheiras & $1(14,2)$ \\
Prurido & $1(8,3)$ & Acne & $1(14,2)$ \\
Hipercromia no local da cicatriz cirúrgica & $1(8,3)$ & Dermatite Atópica & $1(14,2)$ \\
Dermatite seborreica & $1(8,3)$ & & \\
Estrias & $1(8,3)$ & & \\
\hline
\end{tabular}

Tabela 3 - Alterações dermatocosmiátricas desencadeadas pelo tipo de tratamento oncológico

\begin{tabular}{|c|c|c|c|c|c|}
\hline Alterações desencadeadas por cirurgia & $\begin{array}{c}\text { Frequência } \\
\%\end{array}$ & $\begin{array}{l}\text { Alterações desencadeadas pelo } \\
\text { tratamento quimioterápico }\end{array}$ & $\begin{array}{l}\text { Frequência } \\
\%\end{array}$ & $\begin{array}{l}\text { Alterações dermatocosmiátricas } \\
\text { desencadeadas pela radioterapia }\end{array}$ & $\begin{array}{l}\text { Frequência } \\
\%\end{array}$ \\
\hline Linfedema & 32,0 & Eflúvio telógeno/anágeno/alopecia & 28,0 & Radiodermite & 54,0 \\
\hline Eflúvio telógeno/anágeno/alopecia & 14,0 & Xerose cutânea & 25,0 & Xerose cutânea & 25,0 \\
\hline Pilificação na área de reconstrução & 14,0 & Alteração da cor das unhas & 8,0 & Queimadura pós-radioterapia & 5,7 \\
\hline Candidíase & 11,0 & Rarefação da pilificação do corpo & 5,0 & Queilite & 5,7 \\
\hline Limitação do movimento dos mmss & 11,0 & Queilite & 4,8 & Linfedema & 2,8 \\
\hline Granuloma de corpo estranho & 7,0 & Onicólise & 4,3 & Erisipela & 2,8 \\
\hline Escabiose & 4,0 & Candidíase & 3,8 & Alterações mucosas & 2,8 \\
\hline Ausência de mamilo & 4,0 & Rarefação de sobrancelhas & 3,8 & & \\
\hline \multirow[t]{10}{*}{ Hipercromia no local da cicatriz cirúrgica } & 4,0 & Hipercromia pós-quimioterapia & 3,8 & & \\
\hline & & Erisipela & 3,3 & & \\
\hline & & Prurigo & 2,8 & & \\
\hline & & Alteração das mucosas & 2,4 & & \\
\hline & & Dermatite pigmentar linear & 1,4 & & \\
\hline & & Xerostomia & 1,4 & & \\
\hline & & Escabiose & 0,4 & & \\
\hline & & Prurido & 0,4 & & \\
\hline & & Dermatite seborreica & 0,4 & & \\
\hline & & Estrias & 0,4 & & \\
\hline
\end{tabular}


Tabela 4 - Alterações dermatocosmiátricas agravadas em função do tipo de tratamento oncológico

\begin{tabular}{|c|c|c|c|c|c|}
\hline Alterações agravadas por cirurgia & $\begin{array}{l}\text { Frequência } \\
(\%)\end{array}$ & Alterações agravadas por quimioterapia & $\begin{array}{l}\text { Frequência } \\
(\%)\end{array}$ & Alterações agravadas pela radioterapia & $\begin{array}{c}\text { Frequência } \\
(\%)\end{array}$ \\
\hline Cicatrizes inestéticas & 44,0 & Melasma & 31,0 & Dermatite seborreica & 80,0 \\
\hline Onicomicose & 29,0 & Onicmomicose & 20,0 & Pitiríase alba & 20,0 \\
\hline Dermatite seborreica & 8,8 & Tinha das mãos/pés/corpo & 15,0 & & \\
\hline Tinha das mãos/pés/corpo & 5,8 & Dermatite seborreica & 8,7 & & \\
\hline Olheiras & 5,8 & Foliculite & 4,7 & & \\
\hline Foliculite & 2,8 & Verruga Vulgar & 4,7 & & \\
\hline \multirow[t]{6}{*}{ Psoríase } & 2,8 & Paroníquia & 4,7 & & \\
\hline & & Hiperqueratose plantar & 3,9 & & \\
\hline & & Pitiríase alba & 2,3 & & \\
\hline & & Pitiríase versicolor & 0,7 & & \\
\hline & & Acne & 0,7 & & \\
\hline & & Dermatite atópica & 0,7 & & \\
\hline
\end{tabular}

Já as dermatopatias desencadeadas pela radioterapia, apresentaram como manifestações mais frequentes: a radiodermite (54\%), xerose cutânea (25\%), queimadura pós-radioterapia e queilite (5,7\%), linfedema, erisipela e alterações mucosas (2,8\%).

O tipo de tratamento oncológico realizado também interferiu em algumas dermatopatias prévias ao seu início, agravando o quadro (Tabela 4). A cirurgia para excisão do tumor acarretou piora nas lesões cicatriciais inestéticas (44\%), onicomicose (29\%), dermatite seborreica (8,8\%), tinha das mãos/pés/corpo e olheiras (5,8\%), foliculite e psoríse $(2,8)$.

A quimioterapia causou piora das lesões de melasma (31\%), onicomicose (20\%), tinhas das mãos/pés/corpo (15\%), dermatite seborreica $(8,7 \%)$, foliculite, verruga vulgar e paroníquia $(4,7 \%)$, hiperqueratose plantar $(3,9 \%)$, pitiríase alba $(2,3 \%)$, pitiríase versicolor, dermatite atópica e acne $(0,07 \%)$.

Foram agravados pela radioterapia $80 \%$ dos quadros de dermatite seborreica, que intensificou as lesões de pitiríase alba em $20 \%$.

\section{Discussão}

As alterações dermatológicas catalogadas neste ambulatório e por nós descritas são amplamente conhecidas e tratadas em pacientes sadios. Na literatura, já existem relatos das alterações dermatológicas decorrentes do uso de antineoplásicos de forma geral. Nosso estudo visou catalogar as alterações dermatológicas e cosmiátricas decorrentes do tratamento quimioterápico e/ou radioterápico e/ou cirúrgico dos pacientes atendidos no Ambulatório de Reabilitação Dermato-Cosmiátrica da FMABC e, principalmente, separá-las em alterações desencadeadas, agravadas e não relacionadas ao tratamento oncológico, sendo que não encontramos na literatura médica essa forma de subdivisão.

Em nosso estudo, as alterações dermatológicas ou cosmiátricas desencadeadas pelo tratamento oncológico foram mais frequentes que as alterações agravadas e não relacionadas, o que comprova o grande papel que o tratamento quimioterápico e/ou radioterápico e/ou cirúrgico representa nas manifestações cutâneas apresentadas pelos pacientes oncológicos.

A alopecia é a primeira manifestação dermatológica decorrente do uso dos antineoplásicos, cujo aparecimento ocorre logo após o início do tratamento. A severidade da alopecia está relacionada ao número de antineoplásicos utilizados em cada ciclo. Estas drogas causariam tricorrexe, fragmentação, diminuição do diâmetro e despigmentação do fio levando ao quadro de alopecia ${ }^{13}$. Por meio de exames microscópicos, foi demonstrado que os fios afetados pelos antineoplásicos sofrem alterações tanto na pigmentação quanto no seu diâmetro ${ }^{14}$. A perda dos fios ocorre por alterações do ciclo capilar que desencadeia tanto eflúvio telógeno como anágeno simultaneamente, além da fratura dos fios ${ }^{15}$. A alopecia, além de ser a manifestação mais recente, é também a que causa maior impacto na qualidade de vida dos pacientes oncológicos ${ }^{16}$.

A ordem de aparecimento dos sintomas dermatológicos e sua frequência apresentaram poucas variações em determinados estudos. Alguns demonstraram os seguintes efeitos colaterais em ordem de frequência: eflúvio telógeno, despigmentação dos fios capilares, reação eczematosa no local de acesso de administração das drogas, prurido, xerostomia, fenômeno de Raynauld ou livedo, urticária, angioedema, dermatite seborreica, recorrência herpética, pitiríase versicolor, aftas e vitiligo ${ }^{17}$.

Outros apresentaram, em ordem de prevalência, as seguintes manifestações dermatológicas: alopecia (76\%), hiperpigmentação cutânea (31\%), linhas brancas transversas nas unhas (22\%), ictiose (20\%). Outras alterações presentes foram mucosite oral, acne, eritema acral, $f l u$ sing, onicólise, urticária, prurido, flebites e infecções cutâneas ${ }^{18}$.

Somente em um estudo, a alteração capilar não foi o sintoma mais frequente, pois a primeira manifestação dermatológica mais frequente foi rash papulopustular, seguido de hiperpigmentação cutânea, xerose, prurido, queda e alteração da coloração dos fios capilares, alterações periungueais e ungueais e reação palmo-plantar ${ }^{19}$. 
As alterações ungueais descritas são: onicólise ${ }^{20-22}$, alteração da coloração das unhas como hiperpigmentação ${ }^{23,24}$, leuconíquia transversa estriada ou linha de Muehrcke ${ }^{25}$, linhas de Beau ${ }^{26,27}$, hemorragia e abscessos subungueais ${ }^{20}$, onicomadese $\mathrm{e}^{22}$, onicomicose $\mathrm{e}^{28}$ e granuloma piogênico subungueal ${ }^{29}$. A hiperpigmentação ungueal pode se apresentar clinicamente como melanoníquia difusa ${ }^{30}$ ou longitudinal ${ }^{31}$.

As principais alterações cutâneas agudas desencadeadas pelo tratamento radioterápico são: hipopigmentação, telangiectasias, eritema, hiperpigmentação e ulcerações. Os efeitos crônicos são: persistência da hipopigmentação, telangiectasias e ulcerações, fibrose, atrofias, neoplasias malignas ${ }^{8}$ e linfedema ${ }^{32}$.

Neste estudo, observamos que o eflúvio telógeno, anágeno e alopecia representam a primeira manifestação dermatológica desencadeada pelo tratamento oncológico, seguido de xerose, radiodermite, alteração da coloração das unhas, queilite, candidíase, linfedema, onicólise, rarefação de sobrancelhas, erisipela, hipercromia pós-quimioterapia, prurigo, etc.

Portanto, conseguimos catalogar por meio do Ambulatório de Reabilitação Dermato-Cosmiátrica da FMABC, de forma precisa, as alterações dermatológicas decorrentes dos tratamentos oncológicos - quimio e/ou radioterápico e/ou cirúrgico e tratá-las durante esses tratamentos, sem interferir no tratamento oncológico proposto, seja ele quimio, radioterápico ou cirúrgico.

\section{Agradecimento}

Agradecemos a colaboração do Prof. Auro Del Giglio da disciplina de Oncologia da FMABC e dos Profs. Carlos D'Apparecida Santos Machado Filho, Luiz Henrique de Camargo Paschoal e Francisco Paschoal da disciplina de Dermatologia da FMABC.

\section{Referências}

1. Piérard-Frachimont C, Piérard GE. Comment j'explore... une perte de cheveux chez un patient cancéreux. Rev Med Liege 2004;59(9):525-9.

2. Hinds G, Thomas VD. Malignancy and cancer treatment-related hair and nail changes. Dermatol Clin 2008;26(1):59-68.

3. Bursztejn AC, Tréchot $P$, Cuny JF, Schmutz JL, Barbaud A. Cutaneous adverse drug reactions during chemotherapy: consider non-antineoplasic drus. Contact Dermatitis 2008;58(6):365-8.

4. Alley E, Green R, Schulter L. Cutaneous toxicities of cancer therapy. Curr Opin Oncol 2002;14(2):212-6

5. Payne AS, James WD, Weiss RB. Dermatologic toxicity of chemotherapeutic agents. Semin Oncol 2006;33(1):86-97.

6. Mori 0, Matsuo K, Hashimoto T. Anticancer drugs induce apoptosis in mouse hair follicles. Kurume Med 2000:47(3):193-7.

7. Chun YS, Chang SN, Oh D, Park WH. A case of cutaneous reaction to chemotherapeutic agents showing epidermal dysmaturation. J Am Acad Dermatol 2000;43(2 Pt 2):358-60.

8. Koppel RA, Boh EE. Cutaneous reactions to chemotherapeutic agents. Am J Med Sci 2001;321(5):327-35.

9. Mays SR, Cohen PR. Emerging dermatologic issues in the oncology patient. Semin Cutan Med Surg 2006;25(4):179-89.

10. Rupprecht R, Lippold A, Auras C, Bramkamp G, Breitkopf C, Elsmann HJ et al. Late side-effects with cosmetic relevance following soft $X$-ray therapy of cutaneous neoplasias. J Eur Acad Dermatol Venereol 2007;21(2):178-85.

11. Pezzano $M$, Duterque $M$, Lardoux $H$, Louvard $Y$, Morice $M C$, Lefèvre T et al. Radiodermite thoracique en cardiologie interventionnelle. A propos de 6 cas. Arch Mal Couer Vaiss 1999;92(9):1197-204.
12. Fox LP. Pathology and management of dermatologic toxicities associated with anti-EGFR therapy. Oncology (Williston Park) 2006;20(5 Suppl 2):2634.

13. Pai GS, Vilama AM, Dinesh M. Occurrence and severity of alopecia in patients on combination chemotherapy. Indian J Cancer 2000;37(2-3):95-104.

14. Routhouska S, Gilliam AC, Mirmirani P. Hair depigmentation during chemotherapy with a class III/IV receptor tyrosine kinase inhibitor. Arch Dermatol 2006;142(11):1477-9

15. Bleiker TO, Nicolaou N, Traulsen J, Hutchinson PE. 'Atrophic telogen effluvium' from cytotoxic drugs and a randomized controlled trial to investigate the possible protective effect of pretreatment with a topical vitamin D analogue in humans. Br J Dermatol 2005;153(1):103-12.

16. Dorr VJ. A practitioner's guide to cancer-related alopecia. Semin Oncol 1998;25(5):562-70

17. Guillot B, Blazquez L, Bessis D, Dereure 0, Guilhou JJ. A prospective study of cutaneous adverse events induced by low-dose alpha-interferon treatment for malignant melanoma. Dermatology 2004;208(1):49-54.

18. Chiewchanvit S, Noppakun K, Kanchanarattanakon K. Mucocutaneous complications of chemotherapy in 74 patients from Maharaj Nakorn Chiang Mai Hospital. J Med Assoc Thai 2004;87(5):508-14.

19. Lacouture ME, Boerner SA, Lorusso PM. Non-rash skin toxicities associated with targeted therapies. Clin Lung Cancer 2006;8 Suppl 1:S36-42.

20. Ghetti E, Piraccini BM, Tosti A. Onycholysis and subungual haemorrhages secondary to systemic chemotherapy (paclitaxel). J Eur Acad Dermatol Venereol 2003;17(4):459-60.

21. Mackay-Wiggan J, Nair KG, Halasz CL. Onycholysis associated with paclitaxel. Cutis 2003;71(3):229-32. 
22. Chen GY, Chen YH, Hsu MM, Tsao CJ, Chen WC. Onychomadesis and onycholysis associated with capecitabine. Br J Dermatol 2001;145(3):521-2.

23. Prabhash K, Biswas G, Prasad N, Karant N, Sastry PS, Parikh PM. Imatinibinduced nail hyperpigmentation in chronic myeloid leukemia. Indian J Dermatol Venereol Leprol 2006;72(1):63-4.

24. Ghoshal UC, Saha J, Ghoshal U, Ray BK, Santra A, Naik S et al. Pigmented nails and Strongyloides stercoralis infestation causing clinical worsening in a patient treated for immunoproliferative small intestinal disease: two unusual observations. J Diarrhoeal Dis Res 1999;17(1):43-5.

25. Modesto dos Santos V, Sugai TA, Cezar BF, Vasconcellos de Rezende AC. Transverse leukonychia: a case report. Wets Afr J Med 2005;24(2):181-2.

26. Miyoshi I, Kubola T, Taguchi H. Beaus's lines after chemotherapy for ALL. Intern Med 2007;46(1):61.

27. Reissing $\mathrm{A}$, Hengst $\mathrm{U}$, Kroegel $\mathrm{C}$. Beau's lines and chemotherapy. Med Klin (Munich) 2001:96(2):114-5.
28. Cuétara MS, Alhambra A, Moreno JM, Postigo C, Moragues MD, Pontón $\mathrm{J}$ et al. Invasive aspergillosis due to subungual onychomycosis during treatment for non-Hodgkin lymphoma. $\mathrm{Br} J$ Dermatol 2006;154(6):1200-2.

29. Devillers C, Vanhooteghem O, Henrijean A, Ramaut M, de la Brassinne M. Subungueal pyogenic granuloma secondary to docetaxel therapy. Clin Exp Dermatol 2009:34(2):251-2.

30. Hsu KF, Yang MH, Yu JC. Chemotherapy-induced diffuse melanonychia Acta Clin Belg 2008;63(6):427.

31. Saraceno R, Teoli M, Chimenti S. Hydroxyurea associated with concomitant occurrence of diffuse longitudinal melanonychia and multiple squamous cell carcinomas in an elderly subject. Clin Ther 2008;30(7):1324-9.

32. Montero A, Hervás A, Morera R, Sancho S, Córdoba S, Corona JA et al. Control de sintomas crônicos. Efects secundários del tratamiento con radioterapia y quimioterapia. Oncologia (Barc.) 2005;28(3):41-50. 